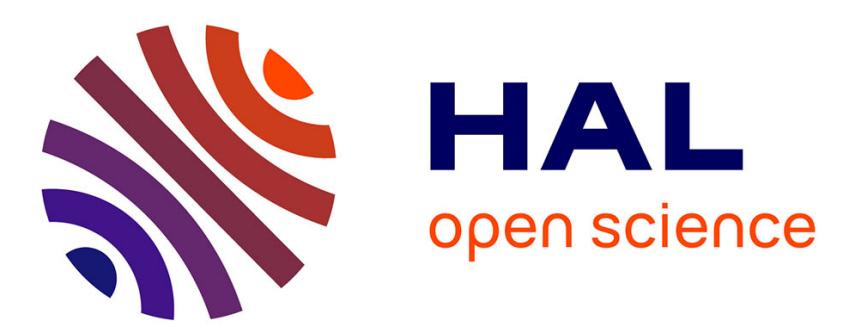

\title{
Structural rigidity optimization with an initial design dependent stress field. Application to thermo-elastic stress loads.
}

\author{
Boris Desmorat
}

\section{- To cite this version:}

Boris Desmorat. Structural rigidity optimization with an initial design dependent stress field. Application to thermo-elastic stress loads.. European Journal of Mechanics - A/Solids, 2013, 37, pp.150 159. 10.1016/j.euromechsol.2012.06.004 . hal-01455321

\section{HAL Id: hal-01455321 \\ https://hal.sorbonne-universite.fr/hal-01455321}

Submitted on 9 Feb 2017

HAL is a multi-disciplinary open access archive for the deposit and dissemination of scientific research documents, whether they are published or not. The documents may come from teaching and research institutions in France or abroad, or from public or private research centers.
L'archive ouverte pluridisciplinaire HAL, est destinée au dépôt et à la diffusion de documents scientifiques de niveau recherche, publiés ou non, émanant des établissements d'enseignement et de recherche français ou étrangers, des laboratoires publics ou privés. 


\title{
Structural rigidity optimization with an initial design dependent stress field. Application to thermo-elastic stress loads.
}

\author{
B. Desmorat \\ UPMC Univ Paris 6, CNRS UMR 7190, Institut Jean Le Rond d'Alembert, F-75005 \\ Paris, France. \\ Univ Paris-Sud, F-91405 Orsay, France.
}

\begin{abstract}
This paper presents a compliance optimization methodology considering an initial stress field. Assuming a local dependency of the initial stress with the optimization parameters, the obtained numerical procedure is composed of finite element stress calculations and local minimization problems that are solved analytically or using a simple bisection procedure. This optimization algorithm is then found to be numerically very efficient. Two optimization problems that fall in the scope of the proposed methodology are considered: topology optimization of the isotropic mixture of two isotropic linear elastic materials (one of the material possibly being void) and orientational optimization (i.e. distribution of anisotropy) with an orthotropic linear elastic material. Numerical examples illustrating the optimization methodology in the case of thermo-elastic stress loads are presented.
\end{abstract}

Keywords: Structural Optimization, Compliance, Thermo-elasticity

Email address: boris.desmorat@upmc.fr () 


\section{Introduction}

In the last two decades, topology optimization has been extensively used and generalized to design dependent loads, such as transmissible sliding force, body load and surface pressure load. A review of such optimizations can be found in Gao and Zhang (2010) and references therein.

An initial stress field is an other class of design dependent load that arise in many mechanical fields (e.g. thermo-elastic stress load in weakly coupled thermo-elasticity, mechanically prestressed structures or residual prestress arising because of manufacturing processes). In the field of thermo-elastic stress loads that will be considered as an application of the proposed optimization methodology, different authors developed different kind of thermoelastic compliance optimization procedures. Following Bendsøe and Kikuchi (1988), Rodrigues and Fernandes (1995) incorporated the thermal effects in the topology design by using the introduction of a material distribution model within a periodic micro-structure in an optimization algorithm based on optimality conditions. Jog (1996) considered distributed parameters topology optimization in the case of non-linear thermo-elasticity using the perimeter method. In such a case, sensitivity analysis techniques were used to formulate stationarity conditions. Pedersen $(2001,2002)$ considered partial compliance (i.e. the part related to the external forces, without the thermo-elastic stress load) to perform topology optimization of plates with prestress using the method of moving asymptotes (MMA) and topology optimization of laminated plates with prestress using a Newton-Raphson procedure. More recently, Pedersen and Pedersen (2010) derived the local compliance sensitivity analysis using its link to strain energy density and defined two thermoelastic 
compliance optimization methodology: one based on optimality criterion for compliance and a second one based on recursive iterations for uniform energy density which is close to fullfill strength maximization. An other optimization strategy was developed by Cho and Choi (2005) in order to take into account the varying in space temperature field (for weakly coupled thermo-elasticity) using an adjoint sensitivity analysis method. Gao and Zhang (2010) considered topology optimization involving thermo-elastic stress loads. They developed a sensitivity analysis of the thermo-elastic compliance in the case of a steady state heat conduction and used it within the method of diagonal quadratic approximation (MDQA). For completeness, we refer also to the work of Li et al. (2001) which considered an other optimization criterion than the thermo-elastic compliance (an evolutionary optimization procedure based on the fully stress design criterion was used to take into account the varying in time temperature field effects on the topology design) and to the work of Sigmund and Torquato (1997) which propose a topology optimization methodology using sequential linear programming that allows to find the distribution of material phases that optimizes some specific thermo-elastic properties, such as zero, negative or maximum thermal expansion.

On the other hand, in the case of linear elasticity, two different approaches of compliance minimization coexist, depending on the choice of a stress based formulation (Allaire, 2002) or a strain based formulation (Bendsøe, 1995). Using the energy theorems, an iterative optimization algorithm which combines local minimizations for the stress based formulation (or local maximizations for the strain based formulation) and global minimizations is defined.

In section 2, we show how to extend the stress based formulation of com- 
pliance optimization with an initial stress field. Considering linearised elasticity, we formulate a relationship between the compliance and the complementary energy in the presence of an initial stress field (section 2.1). The compliance minimization problem with an initial stress field is put into a double minimization problem of a positive quantity with respect to the total stress field and the optimization parameters (section 2.2). Assuming that the initial stress field depends locally on the distributed optimization parameters, we extend the optimization algorithm introduced by Allaire et al. $(1993,1997)$ (which consists in iterative global minimization with respect to the total stress with fixed optimization parameters and local minimizations with respect to optimization parameters with a fixed total stress) to the case of linear elasticity with a design dependent initial stress field (section 2.3).

In sections 3 and 4 , we show how to solve analytically or using a simple bisection procedure the local minimization of elastic energy (with an initial stress field) with respect to the optimization parameters with a fixed total stress field in two important cases:

- topology optimization of the isotropic mixture of two isotropic linear elastic materials, one of the material possibly being void (section 3),

- orientational optimization (i.e. distribution of anisotropy) with an orthotropic linear elastic material (section 4).

This optimization methodology leads to a high numerical efficiency of the numerical procedure, in which one iterates finite elements calculations of stresses and local minimizations which are numerically cost free.

Numerical examples in the case of a thermo-elastic stress load validating 
the proposed optimization procedure in the two previous cases are presented in section 3.4 and 4.3 .

\section{Optimization methodology}

\subsection{Elasticity problem}

We introduce in this section the elasticity problem with an initial stress field, in order to derive the variational formulations and the relation between the compliance and the complementary energy that will be necessary for the definition of the optimization problem.

\subsubsection{Constitutive equations}

Notations used in this paper are the following: bold indicates vectors (e.g. displacement $\boldsymbol{u}$ ), one underbar indicates second order tensors (e.g. strain $\underline{\epsilon}$, stress $\underline{\sigma}$ and initial stress $\left.\underline{\sigma}_{i n i}\right)$ and a double underbar indicates fourth order tensors (e.g. stiffness tensor $\underline{\underline{a}}$ and compliance tensor $\underline{\underline{A}}$ ).

Considering a $3 \mathrm{D}$ medium $\Omega$, the external boundary is split into two surfaces $\Gamma_{0}$ and $\Gamma_{1}$. On $\Gamma_{0}$ is imposed a zero displacement, on $\Gamma_{1}$ a surface load $\boldsymbol{F}$ and inside $\Omega$ a volume load $\boldsymbol{f}$. We make the assumption of small strains and small displacements.

The constitutive equations are:

Equilibrium.

$$
\operatorname{div} \underline{\sigma}+\boldsymbol{f}=0 \quad \text { in } \Omega
$$

Boundary conditions in terms of displacements.

$$
\boldsymbol{u}=0 \quad \text { on } \Gamma_{0}
$$


Boundary conditions in terms of forces.

$$
\underline{\sigma} \boldsymbol{n}=\boldsymbol{F} \quad \text { on } \Gamma_{1}
$$

The initial stress field $\underline{\sigma}_{i n i}$ is introduced in the behaviour law:

$$
\underline{\sigma}=\underline{\underline{a}}: \underline{\epsilon}+\underline{\sigma}_{i n i} \quad \text { with } \epsilon_{i j}=\frac{1}{2}\left(u_{i, j}+u_{j, i}\right)
$$

The elasticity problem $(P)$ of a structure with an initial stress field is defined with the equations (1)-(4).

\subsubsection{Variational formulations}

We introduce in this section the variational formulations used to define the optimization methodology. The key points for the optimization problem formulation are the complementary energy theorem and the relation between the compliance and the complementary energy.

Variational formulation in terms of displacements. If $\boldsymbol{u}$ is solution of the problem $(P)$ then

$$
\begin{gathered}
\left\{\begin{array}{l}
\boldsymbol{u} \in U_{a d} \\
\mathfrak{a}(\boldsymbol{u}, \boldsymbol{v})=L(\boldsymbol{v}) \quad \forall \boldsymbol{v} \in U_{a d}
\end{array}\right. \\
\text { with }\left\{\begin{aligned}
\mathfrak{a}(\boldsymbol{u}, \boldsymbol{v})=\int_{\Omega} \underline{\epsilon}(\boldsymbol{u}): \underline{\underline{a}}: \underline{\epsilon}(\boldsymbol{v}) d V \\
L(\boldsymbol{v})=\int_{\Omega} \boldsymbol{f} \cdot \boldsymbol{v} d V+\int_{\Gamma_{1}} \boldsymbol{F} \cdot \boldsymbol{v} d S-\int_{\Omega} \underline{\sigma}_{i n i}: \underline{\epsilon}(\boldsymbol{v}) d V
\end{aligned}\right.
\end{gathered}
$$

in which $U_{a d}=\left\{\boldsymbol{v} \mid \boldsymbol{v}=\left(v_{1}, v_{2}, v_{3}\right) ; \boldsymbol{v}=0\right.$ on $\left.\Gamma_{0}\right\}$ 
Variational formulation in terms of stresses. Let introduce one possible admissible stress tensor $\underline{\tau}_{0} \in \Sigma_{a d}$. If $\underline{\sigma}$ is solution of the problem $(P)$ then

$$
\begin{gathered}
\qquad\left\{\begin{array}{l}
\underline{\sigma} \in \Sigma_{a d} \\
\mathcal{A}\left(\underline{\sigma}, \underline{\tau}-\underline{\tau}_{0}\right)=\mathcal{L}\left(\underline{\tau}-\underline{\tau}_{0}\right) \quad \forall \underline{\tau} \in \Sigma_{a d}
\end{array}\right. \\
\text { with }\left\{\begin{array}{l}
\mathcal{A}(\underline{\sigma}, \underline{\tau})=\int_{\Omega} \underline{\sigma}: \underline{\underline{A}}: \underline{\tau} d V \\
\mathcal{L}(\underline{\sigma})=\int_{\Omega} \underline{\sigma}: \underline{\underline{A}}: \underline{\sigma}_{i n i} d V
\end{array}\right.
\end{gathered}
$$

in which

$$
\Sigma_{a d}=\left\{\tau_{i j}(i, j=1,2,3), \tau_{i j}=\tau_{j i}, \operatorname{div} \underline{\tau}+\boldsymbol{f}=0 \text { in } \Omega, \underline{\tau} \boldsymbol{n}=\boldsymbol{F} \text { on } \Gamma_{1}\right\}
$$

Complementary energy theorem. If $\underline{\sigma}$ is solution of the problem $(P)$ then

$$
\left\{\begin{array}{l}
\underline{\sigma} \in \Sigma_{a d} \\
J(\underline{\sigma}) \leq J(\underline{\tau}) \quad \forall \underline{\tau} \in \Sigma_{a d}
\end{array} \quad \text { with } J(\underline{\sigma})=\frac{1}{2} \mathcal{A}(\underline{\sigma}, \underline{\sigma})-\mathcal{L}(\underline{\sigma})\right.
$$

2.1.3. Relation between the compliance and the complementary energy

The compliance $L(\boldsymbol{u})$ (defined in equation (6)) and the complementary energy $J(\underline{\sigma})$ (defined in equation $(8)$ ) are linked by the relation:

$$
L(\boldsymbol{u})=2 J(\underline{\sigma})+\int_{\Omega} \underline{\sigma}_{i n i}: \underline{\underline{A}}: \underline{\sigma}_{i n i} d V
$$

Proof:

$$
\begin{aligned}
L(\boldsymbol{u})=\mathfrak{a}(\boldsymbol{u}, \boldsymbol{u}) & =\int_{\Omega} \underline{\epsilon}(\boldsymbol{u}): \underline{\underline{a}}: \underline{\epsilon}(\boldsymbol{u}) d V=\int_{\Omega}\left(\underline{\sigma}-\underline{\sigma}_{i n i}\right): \underline{\underline{A}}:\left(\underline{\sigma}-\underline{\sigma}_{i n i}\right) d V \\
& =\left[\int_{\Omega} \underline{\sigma}: \underline{\underline{A}}: \underline{\sigma} d V-2 \int_{\Omega} \underline{\sigma}: \underline{\underline{A}}: \underline{\sigma}_{i n i} d V\right]+\int_{\Omega} \underline{\sigma}_{i n i}: \underline{\underline{A}}: \underline{\sigma}_{i n i} d V
\end{aligned}
$$




\subsection{Optimization problem}

We consider bounded distributed optimization parameters $\beta \in\left[\beta_{\text {min }}, \beta_{\text {max }}\right]$. The compliance is chosen as a measure of the global rigidity of the elastic structure with an initial stress field. In order to limit the total amount of some of the optimization parameters, a cost term is introduced in the definition of the criterion with the help of some positive smooth function $\operatorname{cost}(\beta)$. The optimization problem is written:

$$
\min _{\beta \in\left[\beta_{\min }, \beta_{\max }\right]}\left[\int_{\Omega} \boldsymbol{f} \cdot \boldsymbol{u} d V+\int_{\Gamma_{1}} \boldsymbol{F} . \boldsymbol{u} d S-\int_{\Omega} \underline{\sigma}_{i n i}: \underline{\epsilon}(\boldsymbol{u}) d V+\int_{\Omega} \operatorname{cost}(\beta) d V\right]
$$

Considering the relation (10) between the compliance and the complementary energy and the complementary energy theorem (9), the optimization problem reads:

$$
\min _{\beta \in\left[\beta_{\min }, \beta_{\max }\right]}\left[\min _{\underline{\tau} \in \Sigma_{a d}}\left[2 J(\underline{\tau})+\int_{\Omega} \underline{\sigma}_{i n i}: \underline{\underline{A}}: \underline{\sigma}_{i n i} d V\right]+\int_{\Omega} \operatorname{cost}(\beta) d V\right]
$$

which takes the form:

$$
\min _{\beta \in\left[\beta_{\min }, \beta_{\max }\right]} \min _{\underline{\tau} \in \Sigma_{a d}}\left[2 J(\underline{\tau})+\int_{\Omega} \underline{\sigma}_{i n i}: \underline{\underline{A}}: \underline{\sigma}_{i n i} d V+\int_{\Omega} \operatorname{cost}(\beta) d V\right]
$$

or finally:

$$
\min _{\beta \in\left[\beta_{\min }, \beta_{\max }\right]} \min _{\underline{\tau} \in \Sigma_{a d}} \int_{\Omega}\left[\left(\underline{\tau}-\underline{\sigma}_{i n i}\right): \underline{\underline{A}}:\left(\underline{\tau}-\underline{\sigma}_{i n i}\right)+\operatorname{cost}(\beta)\right] d V
$$

\subsection{Optimization algorithm}

In order to obtain a numerically very efficient optimization algorithm, we are looking forward to a local sensitivity analysis. 
We then assume that at each point of position $\boldsymbol{X}$ in the domain $\Omega$, the initial stress field $\underline{\sigma}_{i n i}$ is only function of the value of the distributed optimization parameters $\beta$ at this point, i.e. $\underline{\sigma}_{i n i}[\beta(\boldsymbol{X})]$. In other words, the initial stress field depends locally on the distributed optimisation parameters.

With this assumption, it is possible to extend the optimization algorithm introduced by Allaire et al. $(1993,1997)$ in the case of classical compliance optimization problem.

The optimization algorithm reads:

(a) Initialisation - Iteration 0 .

- Choice of $\beta(\boldsymbol{X})=\beta^{(0)}$

- Calculation of the initial stress field $\underline{\sigma}_{i n i}\left[\beta^{(0)}\right]=\underline{\sigma}_{i n i}^{(0)}$

- Calculation of the total stress $\underline{\sigma}^{(0)}$

(b) Local minimisations with respect to optimisation parameters with a fixed total stress.

At each point of the structure, the following problem is solved:

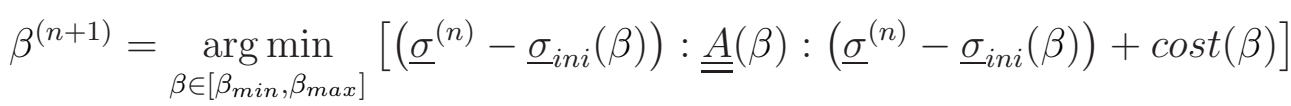

(c) Global minimisation with respect to total stress field with fixed optimisation parameters.

For given optimisation parameters $\beta^{(n+1)}$, the total stress field $\underline{\sigma}^{(n+1)}$ is calculated by solving the elasticity problem (1)-(4). During this step, the complementary energy theorem (9), in which the statically admissible 
stress $\tau$ is chosen equal to the total stress of the previous iteration $\underline{\sigma}^{(n)}$, reads:

$$
J\left(\underline{\sigma}^{(n+1)}\right) \leq J\left(\underline{\sigma}^{(n)}\right)
$$

(d) Back to step (b).

Optimisation algorithm convergence. Integrating on the whole domain, step (b) of the optimisation algorithm gives:

$$
\begin{aligned}
\int_{\Omega}\left[\left(\underline{\sigma}^{(n)}-\underline{\sigma}_{i n i}^{(n+1)}\right): \underline{\underline{A}}^{(n+1)}:\left(\underline{\sigma}^{(n)}-\underline{\sigma}_{i n i}^{(n+1)}\right)+\operatorname{cost}^{(n+1)}\right] d V \\
\leq \int_{\Omega}\left[\left(\underline{\sigma}^{(n)}-\underline{\sigma}_{i n i}^{(n)}\right): \underline{\underline{A}}^{(n)}:\left(\underline{\sigma}^{(n)}-\underline{\sigma}_{i n i}^{(n)}\right)+\operatorname{cost}^{(n)}\right] d V
\end{aligned}
$$

Adding the term $\int_{\Omega} \underline{\underline{\sigma}}_{i n i}^{(n+1)}: \underline{\underline{A}}^{(n+1)}: \underline{\sigma}_{i n i}^{(n+1)} d V$ to (16) gives :

$$
\begin{aligned}
\int_{\Omega}\left[\left(\underline{\sigma}^{(n+1)}-\underline{\sigma}_{i n i}^{(n+1)}\right): \underline{\underline{A}}^{(n+1)}:\left(\underline{\sigma}^{(n+1)}-\underline{\sigma}_{i n i}^{(n+1)}\right)+\operatorname{cost}^{(n+1)}\right] d V \\
\quad \leq \int_{\Omega}\left[\left(\underline{\sigma}^{(n)}-\underline{\sigma}_{i n i}^{(n+1)}\right): \underline{\underline{A}}^{(n+1)}:\left(\underline{\sigma}^{(n)}-\underline{\sigma}_{i n i}^{(n+1)}\right)+\operatorname{cost}^{(n+1)}\right] d V
\end{aligned}
$$

Considering (17) and (18), we get:

$$
\begin{aligned}
\int_{\Omega}\left[\left(\underline{\sigma}^{(n+1)}-\underline{\sigma}_{i n i}^{(n+1)}\right): \underline{\underline{A}}^{(n+1)}:\left(\underline{\sigma}^{(n+1)}-\underline{\sigma}_{i n i}^{(n+1)}\right)+\operatorname{cost}^{(n+1)}\right] d V \\
\quad \leq \int_{\Omega}\left[\left(\underline{\sigma}^{(n)}-\underline{\sigma}_{i n i}^{(n)}\right): \underline{\underline{A}}^{(n)}:\left(\underline{\sigma}^{(n)}-\underline{\sigma}_{i n i}^{(n)}\right)+\operatorname{cost}^{(n)}\right] d V
\end{aligned}
$$

The criterion being a positive quantity and decreasing at each iteration (step (b) - step (c)), the algorithm necessarily converges to a limit. This limit is a stationnary point of the criterion and, in numerical practice, a local minimizer. 


\section{Topology optimization with isotropic linear elastic materials with an initial stress field}

\subsection{Dependence of the initial stress with the optimization parameters}

Let consider the mixture of two isotropic materials such that the composite stiffness and dilatation tensors are isotropic. Densities, bulk moduli and shear moduli are respectively noted $\rho, \kappa$ and $\mu$ with subscripts 1,2 and $C$ for material 1, material 2 and composite. In the framework of structural rigidity optimization, we assume that

$$
\rho_{1}<\rho_{2} \quad \kappa_{1}<\kappa_{2} \quad \mu_{1}<\mu_{2}
$$

which implies that a composite density increase leads to a composite stiffness increase.

Using the SIMP method (introduced for topology optimization with material and void (Bendsøe, 1989; Zhou and Rozvany, 1991)) in the case of topology optimization with two distinct bulk materials, the optimization parameter is the fictitious material density $\rho=\frac{\rho_{C}}{\rho_{2}}$, which varies in the range $\left[\rho_{\min }, 1\right]$, with $\rho_{\min }=\frac{\rho_{1}}{\rho_{2}}$. The isotropic composite stiffness tensor $\underline{\underline{a}}$ is considered proportional to the stiffness tensor $\underline{\underline{a}}_{0}$ chosen equal to the stiffness tensor of material 2:

$$
\underline{\underline{a}}=\rho^{n} \underline{\underline{a}}_{0} \quad \text { with } \quad\left(a_{0}\right)_{i j k l}=\kappa_{2} \delta_{i j} \delta_{k l}+\mu_{2}\left(\delta_{i k} \delta_{j l}+\delta_{j k} \delta_{i l}-\frac{2}{3} \delta_{i j} \delta_{k l}\right)
$$

Assumption (20) implies that $\mathrm{n}$ is strictly positive. The phenomenological power law approximation (21) of the composite bulk and shear moduli with the same exponent $n$ may be considered for the mixture of two materials with close shear and bulk moduli ratios $\left(\frac{\mu_{1}}{\mu_{2}} \approx \frac{\kappa_{1}}{\kappa_{2}}\right)$, i.e. with close poisson's ratio, 
or for the mixture of material and void (other more accurate approximations such as the RAMP or the modified SIMP approaches are not considered in the present work). If material 1 degenerates to void, the density $\rho_{1}$, bulk modulus $\kappa_{1}$ and shear modulus $\mu_{1}$ tends to zero, and a very small positive value of $\rho_{\text {min }}$ is chosen for numerical purposes. In this case, the coefficient $n$ may be experimentally determined (e.g. $n \approx 2$ for open cell metal foams (Ashby, 2006)) or may be introduced to limit the amount of intermediate densities when no design dependent initial stress is considered (and is then classically chosen equal to 3$)$.

The optimization methodology applies to problems with a local dependency of the initial stress with the optimization parameters (see section 2.3).

The initial stress depends on the optimization parameters through the elasticity tensor:

$$
\underline{\sigma}_{i n i}=\underline{\underline{a}}: \underline{\epsilon}_{i n i}(\boldsymbol{X})
$$

The dependency of the initial strain field $\underline{\epsilon}_{i n i}$ with the density $\rho$ is arbitrarily considered in the form:

$$
\underline{\epsilon}_{i n i}=\rho^{p} \underline{\epsilon}_{0}
$$

in which $\underline{\epsilon}_{0}$ is independent of the the density $\rho$.

For the topology optimization with an initial stress, we will then consider an initial stress in the form

$$
\underline{\sigma}_{i n i}=\rho^{n+p} \underline{\sigma}_{0} \quad(\text { with } \quad n>0)
$$

in which $\underline{\sigma}_{0}=\underline{a}_{0}: \underline{\epsilon}_{0}$ is independent of $\rho$.

As it will be exemplified in section (3.3) in the thermo-elasticity framework, the parameter $p$ can take values which are positive or negative, de- 
pending on which materials are mixed.

\subsection{Local minimizations}

In the case of an initial stress depending on the optimization parameter through equation (24), the local criterion defined in (14) reads:

$$
\begin{aligned}
\operatorname{crit}(\rho) & =\underline{\sigma}: \underline{\underline{A}}: \underline{\sigma}+\underline{\sigma}_{i n i}: \underline{\underline{A}}: \underline{\sigma}_{i n i}-2 \underline{\sigma}: \underline{\underline{A}}: \underline{\sigma}_{i n i}+\operatorname{cost}(\rho) \\
& =\frac{1}{\rho^{n}} \underline{\sigma}: \underline{\underline{A}}_{0}: \underline{\sigma}+\rho^{n+2 p} \underline{\sigma}_{0}: \underline{\underline{A}}_{0}: \underline{\sigma}_{0}-2 \rho^{p} \underline{\sigma}: \underline{\underline{A}}_{0}: \underline{\sigma}_{0}+\operatorname{cost}(\rho)
\end{aligned}
$$

The local minimization (with a fixed total stress field) problem reads then:

$$
\min _{\rho \in\left[\rho_{\text {min }}, 1\right]}\left[\frac{B}{\rho^{n}}+\rho^{n+2 p} B_{0}-2 \rho^{p} C+\operatorname{cost}(\rho)\right] \quad \text { with }\left\{\begin{array}{c}
B=\underline{\sigma}: \underline{A}_{0}: \underline{\sigma} \\
B_{0}=\underline{\sigma}_{0}: \underline{\underline{A}}_{0}: \underline{\sigma}_{0} \\
C=\underline{\sigma}: \underline{\underline{A}}_{0}: \underline{\sigma}_{0}
\end{array}\right.
$$

The cost functional is chosen proportional to the distributed fictitious density: $\operatorname{cost}(\rho)=k \rho$, where the parameter $k$ is a positive constant chosen by the user.

The second derivative of the local criterion with respect to the density $\rho$ reads:

$\frac{\partial^{2} \operatorname{crit}(\rho)}{\partial \rho^{2}}=\frac{1}{\rho^{n+2}}\left[(n+2 p)(n+2 p-1) B_{0} \rho^{2(n+p)}-2 p(p-1) C \rho^{n+p}+n(n+1) B\right]$

Considering that $C^{2}-B B_{0} \leq 0, B \geq 0, B_{0} \geq 0$ and $n>0$, the second derivative of the local criterion is always positive (i.e. independently of the stress state) if and only if

$$
p_{\text {min }} \leq p \leq p_{\max } \quad \text { with }\left\{\begin{array}{l}
p_{\min }=n+1-\sqrt{2 n(n+1)} \\
p_{\max }=n+1+\sqrt{2 n(n+1)}
\end{array}\right.
$$


Remark: $n<p_{\max }$ for all $n, p_{\min }<n$ for $n>(\sqrt{3}-1) / 2$ and $p_{\min }<0$ for $n>1$.

The first derivative of the local criterion reads:

$$
\frac{\partial \operatorname{crit}(\rho)}{\partial \rho}=\frac{1}{\rho^{n+1}}\left[(n+2 p) B_{0} \rho^{2(n+p)}-2 p C \rho^{n+p}-n B+k \rho^{n+1}\right]
$$

With $p$ satisfying eq (27), the first derivative is monotonic increasing on ] $0,+\infty\left[\right.$ starting from negative values, and is equal to zero for $\rho_{0}$ solution of

$$
(n+2 p) B_{0} \rho^{2(n+p)}-2 p C \rho^{n+p}+k \rho^{n+1}-n B=0
$$

Let $\rho_{\text {opt }}$ be the point for which $\operatorname{crit}(\rho)$ is minimum for $\left.\left.\rho \in\right] 0,1\right]$. Then:

$$
\rho_{\text {opt }}=\min \left(1, \rho_{0}\right)
$$

In order to find numerically the optimal density value, equation (28) is solved using a bisection procedure for any value of $p$ satisfying to equation (27). If $p=1$ (which consists in a linear increasing variation of the initial strain $\underline{\epsilon}_{i n i}$ with respect to the density $\rho$ ), the equation (28) can be analytically solved:

$$
\rho_{0}=\left(\frac{2 C-k+\sqrt{(2 C-k)^{2}+4 n(n+2) B B_{0}}}{2(n+2) B_{0}}\right)^{\frac{1}{n+1}}
$$

\subsection{Mechanical interpretation of the power-law approximation of the initial} strain and stress in the thermo-elasticity framework

Let consider the mixture of two materials 1 and 2 such that the composite stiffness increases with a density increase $\left(\rho_{1}<\rho_{2}, \kappa_{1}<\kappa_{2}, \mu_{1}<\mu_{2}\right.$ and $n>0)$. 
In the presence of thermo-elastic stress load, the initial stress is

$$
\underline{\sigma}_{i n i}=\underline{\underline{a}}: \underline{\epsilon}_{i n i} \quad \text { with } \quad \underline{\epsilon}_{i n i}=-\underline{\alpha} \Delta T
$$

in which $\underline{\underline{a}}$ is the composite isotropic stiffness tensor, $\underline{\alpha}$ is the composite isotropic dilatation tensor and $\Delta T=T-T_{0}$ the variation of temperature with respect to a reference temperature $T_{0}$.

The temperature field is a function of the material density $\rho$ through a thermal problem, and thus depends globally on the material density (the temperature at each point is a function of the distribution of the material density over the entire domain). The initial stress may then depend locally on the material density if and only if the temperature is constant over the entire domain.

Using the SIMP approach (24) in this thermo-elastic framework, the initial stress reads then

$$
\underline{\sigma}_{i n i}=\rho^{n+p} \underline{\sigma}_{0} \quad \text { with } \quad \underline{\sigma}_{0}=-\underline{a}_{0}: \underline{\alpha}_{0} \Delta T
$$

in which the isotropic stiffness tensors $\underline{\underline{a}}_{0}$, the isotropic dilatation tensor $\underline{\alpha}_{0}$ and the temperature $\Delta T$ are independent of the density $\rho$.

We will show in the following part of this section that the values of the thermal expansion coefficients $\alpha_{1}$ and $\alpha_{2}$ of the two mixed materials may lead to positive or negative values of the parameter $p$.

In the following, the numerical values of the parameter $n$ are obtained by fitting simultaneously (assuming that Poisson's ratio of the two bulk materials are close) the effective bulk and shear moduli of the composite defined 
with the Hashin-Shtrikman upper bound (Hashin and Shtrikman, 1963):

$$
\kappa_{C}=\kappa_{2}+\frac{V_{1}}{\frac{1}{\kappa_{1}-\kappa_{2}}+\frac{3 V_{2}}{3 \kappa_{2}+4 \mu_{2}}} \quad \mu_{C}=\mu_{2}+\frac{V_{1}}{\frac{1}{\mu_{1}-\mu_{2}}+\frac{6\left(\kappa_{2}+2 \mu_{2}\right) V_{2}}{5 \mu_{2}\left(3 \kappa_{2}+4 \mu_{2}\right)}}
$$

in which the volume fractions $V_{1}$ and $V_{2}$ of materials 1 and $2\left(\right.$ with $V_{1}+V_{2}=1$ ) are related to the fictitious density $\rho$ by

$$
\rho=V_{1} \rho_{\min }+V_{2} \quad\left(\text { with } \quad \rho_{\min }=\frac{\rho_{1}}{\rho_{2}}\right)
$$

The numerical values of $p$ are obtained by fitting the effective coefficient of thermal expansion of the composite explicitly given in terms of the effective bulk modulus (Levin, 1967):

$$
\alpha_{C}=V_{1} \alpha_{1}+V_{2} \alpha_{2}+\frac{\alpha_{1}-\alpha_{2}}{\frac{1}{\kappa_{1}}-\frac{1}{\kappa_{2}}}\left[\frac{1}{\kappa_{C}}-\left(\frac{V_{1}}{\kappa_{1}}+\frac{V_{2}}{\kappa_{2}}\right)\right]
$$

The values of $p$ may be classified in five different cases (the material parameters used for the numerical examples are presented in table 1):

Case 1: $p>0$. A density increase of the composite leads to an increase of the thermal dilatation and an increase of the initial stress. Example: titanium/copper $(n=0.2$ and $p=0.9)$.

Case 2: $p=0$. A density increase of the composite leads to a constant thermal dilatation and a increase of the initial stress. This case may be obtained with the mixture of two materials with the same thermal expansion coefficient, or in the case of material and void (for which the very small value 
of void stiffness implies that the composite thermal expansion coefficient is equal to the material 2 thermal expansion coefficient).

Case 3: $-n<p<0$. A density increase of the composite leads to a decrease of the thermal dilatation and a increase of the initial stress. Example: aluminium/copper $(n=0.6$ and $p=-0.3)$.

Case 4: $p=-n$. A density increase of the composite leads to a decrease of the thermal dilatation and a constant initial stress.

Case 5: $p<-n$. A density increase of the composite leads to a decrease of the thermal dilatation and a decrease of the initial stress. Example: aluminium/titanium $(n=1.0$ and $p=-1.9)$.

In this thermo-elasticity framework, the optimization methodology applicability condition (27) includes the case 2 and partially the cases 1 and 3 .

\subsection{Numerical example in the case of a thermo-elastic stress load}

We consider topology optimization of material and void. The parameter $\mathrm{p}$ is then equal to zero (as explained in the previous section (3.3)). The value of $n$ is chosen equal to 3, i.e.

$$
\underline{\sigma}_{i n i}=\rho^{3} \underline{\sigma}_{0} \quad \text { with } \quad \underline{\sigma}_{0}=-\underline{\underline{a}}_{0}: \underline{\alpha}_{0} \Delta T
$$


in which $\underline{a}_{0}$ and $\underline{\alpha}_{0}$ are the isotropic stiffness and dilatation tensors of the bulk material. With the chosen values of $n$ and $p$, the condition (27) is satisfied and the local minimization is solved using a bisection method.

The numerical example consists in a 3D beam with dimensions 1x1x4. A vertical force is applied at the center of a long face while the two ends are clamped (see figure 1). We will consider two thermo-elastic stress load cases: no thermo-elastic stress load and a thermo-elastic stress load induced by a constant temperature of $-50^{\circ} \mathrm{C}$.

The isotropic material considered is steel with elasticity and thermal parameters defined in table 2 .

The initial optimization parameters (density $\rho$ ) values are fixed to 1 . The optimization algorithm used is described in section 2.3. Convergence is obtained for a relative error equal to $0.01 \%$. The optimal distributions in the two loading cases fulfil the property of $20 \%$ of total volume used (either by iterating over the entire optimization algorithm with updated values of the cost parameter $k$ or by iterating over the local minimizations adjusting the value of the cost parameter $k$ as described by Allaire (2002)).

To avoid mesh sensitivity, the numerical approach of non-local energy density in the local minimization step is used (Desmorat, 2007).

Initial and optimal values of compliance and maximal displacement are presented in table 3. Without thermo-elastic stress load, the initial compliance is smaller than the optimal compliance because of the initial density equal to 1 in the entire domain. This is no longer true with thermo-elastic stress load, the load depending on the density.

The optimal density distribution without thermo-elastic stress loads is 
presented in figure 2. This distribution reaches a clear black and white design (with "grey" regions only present in the transition from "black" to "white" regions, which is due to the numerical approach of non-local energy density in the local minimization step and can be removed by iterating a few more iterations with a local energy density approach).

The optimal density distribution with thermo-elastic stress loads is presented in figure 3. This optimal distribution of density is no longer close to 0 and 1 as in the case without thermo-elastic stress load, as already noticed by Rodrigues and Fernandes (1995).

We remark in those figures that the topology is completely different when the thermo-elastic stress load is added. If the loading with thermal loads is applied to the optimal design obtained without thermal loads, the compliance and the maximal displacements are greater than those obtained on the optimal designs obtained with thermal loads, even though the latter is not a black and white design.

In order to penalize the optimal density distribution, it is possible to use a penalized density $\rho_{\text {pen }}$ which is closer to 0 for $\rho_{\text {opt }}<1 / 2$ or 1 for $\rho_{\text {opt }}>1 / 2$ in the local minimization of the energy density. The penalized optimal density used is:

$$
\begin{aligned}
\text { if } \rho_{\text {opt }} & \leq \frac{1}{2}: & \rho_{\text {pen }} & =\frac{1-e^{\lambda \rho_{\text {opt }}}}{2\left(1-e^{\lambda / 2}\right)} \\
\text { if } \rho_{\text {opt }} & \geq \frac{1}{2}: & \rho_{\text {pen }} & =\frac{1}{2}+\frac{1-e^{-\lambda\left(\rho_{o p t}-\frac{1}{2}\right)}}{2\left(1-e^{-\lambda / 2}\right)}
\end{aligned}
$$

A greater value of the parameter $\lambda$ leads to a penalized density closer to 0 or 1 (with $\lim _{\lambda \rightarrow 0} \rho_{\text {pen }}=\rho_{\text {opt }}$ ). Only a few iterations need to be performed after convergence with a fixed parameter $k$ equal to the value obtained at 
convergence to obtain a relative error of $0.01 \%$ on the compliance and the total material volume. With $\lambda=5.5$, the total material volume obtained after the penalization process is equal to $20.0 \%$, and the penalized optimal density distribution is presented in figure 4 . This penalization procedure leads to a black and white design associated to a $5 \%$ increase of the compliance (and a $24 \%$ decrease of the maximal displacement).

\section{Orientational optimization with an orthotropic linear elastic ma- terial with an initial stress field}

\subsection{Dependence of the initial stress with the optimization parameters}

In this section, we consider 2D elasticity under the assumption of plane stress. The optimization parameter is the orthotropic material orientation $\phi$. For the optimal orientation of an orthotropic material with an initial stress, we assume that the initial stress function of the material orientation $\underline{\sigma}_{i n i}(\phi)$, has its principal directions aligned with the orthotropy principal directions.

This assumption is linked to the assumed local character of the initial stress with respect to the material orientation: in this case we suppose that the initial stress is related to the material symmetries, and consider the simplest case of aligned directions. An example (detailed in section 4.3) is the thermo-elastic behaviour of a material with an orthotropic stiffness tensor and an isotropic dilatation tensor.

The orientation parameter $\phi$ has no influence on the mass of the structure: the cost term introduced in (11) is chosen equal to zero. 


\subsection{Local minimizations}

Considering the local minimizations step (with a fixed total stress $\underline{\sigma}$ ) of the optimization algorithm, the optimal orientation is solution of:

$$
\min _{\phi \in]-\pi / 2, \pi / 2]}\left[\underline{\sigma}: \underline{\underline{A}}(\phi): \underline{\sigma}-2 \underline{\sigma}: \underline{\underline{A}}(\phi): \underline{\sigma}_{i n i}(\phi)+\underline{\sigma}_{i n i}(\phi): \underline{\underline{A}}(\phi): \underline{\sigma}_{i n i}(\phi)\right]
$$

In the case of low shear stiffness material (defined by $c_{2}<0$, see equation (43)), the optimal orientation is found to be aligned with one of the principal stress direction. The choice depends on the initial stress field and on the material parameters.

To prove it, we follow the proof presented by Cheng and Pedersen (1997) in the case of linear elasticity (with no initial stress).

First, we remark that $C_{1}=\underline{\sigma}_{i n i}(\phi): \underline{\underline{A}}(\phi): \underline{\sigma}_{i n i}(\phi)$ does not depend on the material orientation $\phi$, because of the assumption of aligned initial stress principal directions with orthotropy principal direction.

In the orthotropy frame and using the matrix notations,

$$
\underline{\underline{A}}=\left(\begin{array}{ccc}
A_{11} & A_{12} & 0 \\
A_{12} & A_{22} & 0 \\
0 & 0 & A_{33}
\end{array}\right) \quad \underline{\sigma}=\left(\begin{array}{c}
\sigma_{11} \\
\sigma_{22} \\
\sqrt{2} \sigma_{12}
\end{array}\right)
$$

Let assume (without loss of generality) that $A_{11}-A_{22}<0$. Let $\phi$ be the angle between the stiffest direction of the orthotropy frame and the direction of maximal principal stress (in absolute value). The two principal stresses are noted $\sigma_{I}$ and $\sigma_{I I}$, with $\left|\sigma_{I}\right|>\left|\sigma_{I I}\right|$. After some calculations, the local 
criterion reads:

$$
\begin{aligned}
\text { crit } & =\left[\underline{\sigma}: \underline{\underline{A}}(\phi): \underline{\sigma}-2 \underline{\sigma}: \underline{\underline{A}}(\phi): \underline{\sigma}_{i n i}(\phi)+C_{1}\right] \\
& =\left[D_{0}+2 D_{1} \cos (2 \phi)+D_{2} \cos ^{2}(2 \phi)+C_{1}\right]
\end{aligned}
$$

with

$$
\begin{aligned}
D_{0}= & \frac{1}{4}\left(\sigma_{I}^{2}-\sigma_{I I}^{2}\right) 2 A_{33}+\frac{1}{4}\left(\sigma_{I}^{2}+\sigma_{I I}^{2}\right)\left(A_{11}+A_{22}+2 A_{12}\right) \\
& +\left(\sigma_{I}+\sigma_{I I}\right)\left[\left(A_{11}+A_{12}\right) \underline{\sigma}_{11}^{i n i}+\left(A_{12}+A_{22}\right) \underline{\sigma}_{22}^{i n i}\right] \\
D_{1}= & \frac{1}{4}\left(\sigma_{I}^{2}-\sigma_{I I}^{2}\right)\left(A_{11}-A_{22}\right)+\frac{1}{2}\left(\sigma_{I}-\sigma_{I I}\right)\left[\left(A_{11}-A_{12}\right) \underline{\sigma}_{11}^{i n i}+\left(A_{12}-A_{22}\right) \underline{\sigma}_{22}^{i n i}\right] \\
D_{2}= & \frac{1}{4}\left(\sigma_{I}^{2}-\sigma_{I I}^{2}\right)\left(A_{11}+A_{22}-2 A_{12}-2 A_{33}\right)
\end{aligned}
$$

Then we calculate the first derivative of the local criterion, and equal it to zero:

$$
\frac{\partial \text { crit }}{\partial \phi}=0 \Leftrightarrow D_{1} \sin (2 \phi)+D_{2} \sin (2 \phi) \cos (2 \phi)=0 \Leftrightarrow\left\{\begin{array}{l}
\phi=0 \\
\phi=\pi / 2 \\
\cos (2 \phi)=-\zeta
\end{array}\right.
$$

with

$$
\zeta=\frac{c_{0}\left(\sigma_{I}+\sigma_{I I}\right)+c_{1}}{c_{2}\left(\sigma_{I}-\sigma_{I I}\right)} \quad \text { and } \quad\left\{\begin{array}{l}
c_{0}=A_{11}-A_{22} \\
c_{1}=2\left[\left(A_{11}-A_{12}\right) \underline{\sigma}_{11}^{i n i}+\left(A_{12}-A_{22}\right) \underline{\sigma}_{22}^{i n i}\right] \\
c_{2}=A_{11}+A_{22}-2 A_{12}-2 A_{33}
\end{array}\right.
$$

The difference with the elastic case without the initial stress is the term $c_{1}$. Let's now calculate the difference between the different values of the local 
criterion for $\phi=0, \phi=\frac{\pi}{2}$ and $\cos (2 \phi)=-\zeta$ :

$$
\begin{aligned}
& \operatorname{crit}[\phi=0]-\operatorname{crit}[\cos (2 \phi)=-\zeta]=c_{2}\left(\sigma_{I}-\sigma_{I I}\right)^{2}(1+\zeta)^{2} \\
& \operatorname{crit}[\phi=0]-\operatorname{crit}[\phi=\pi / 2]=4 D_{1}
\end{aligned}
$$

In the case of low shear stiffness material $\left(c_{2}<0\right)$, the solution of the local minimization of the criterion is then $\phi=0$ or $\phi=\frac{\pi}{2}$, depending on the sign of $D_{1}$, which is a function of the initial stress field and of the material parameters.

Remark: With no initial stress, $D_{1}$ is always negative, and then $\phi=0$ is the optimal orientation in the case of low shear stiffness material, i.e. the direction of maximal stiffness is aligned with the principal stress direction associated to the principal stress that has maximal absolute value.

\subsection{Numerical example in the case of a thermo-elastic stress load}

The temperature field is a function of the material orientation $\phi$ through a thermal problem, and thus depends globally on the material orientation (the temperature at each point is a function of the distribution of the material orientation over the entire domain). The initial stress may then depend locally on the material orientation if and only if the material orientation has no influence on the thermal conductivity, i.e. that the thermal conductivity is isotropic. With the temperature independent of the material orientation $\phi$, the initial thermo-elastic stress reads:

$$
\underline{\sigma}_{i n i}=-\underline{\underline{a}}(\phi): \underline{\alpha}(\phi) \Delta T
$$

in which $\underline{\underline{a}}$ is the orthotropic stiffness tensor and $\underline{\alpha}$ is the orthotropic dilatation tensor. 
The numerical example consists in a $2 \mathrm{D}$ plate with dimensions $1 \mathrm{x} 1$. The left edge is clamped, temperatures of $-100^{\circ} \mathrm{C}$ and $+100^{\circ} \mathrm{C}$ are respectively imposed on the upper and lower edges and a zero heat flux is imposed on the left and right edges of the plate (see figure 5). The orthotropic material considered is a monolayered composite made of long curvilinear aramid fibres with elasticity and thermal parameters defined in table 4. The initial orthotropy (fibres) orientation is aligned with the horizontal axis in the entire plate.

Because of the isotropic thermal conductivity of such a material, the temperature field is independent of the optimization parameters. The temperature field can then be obtained before the optimization procedure by solving the heat equation: in the present case, the temperature field is linear from the upper edge to the lower edge of the plate (for more complicated boundary conditions, the temperature field should be computed numerically). On the other hand, the orthotropy principal directions in rigidity are aligned with those of thermal dilatation. The optimization algorithm described in section 2.3 can then be used. Convergence is obtained for a relative error equal to $0.01 \%$.

The optimal orthotropy orientation is presented in figure 6. Initial and optimal deformed configuration are presented in figures 7 and 8. At convergence, the decrease of the compliance is $58 \%$ and the decrease of maximal displacement is $16 \%$. 


\section{Conclusion}

In this paper, we propose a double minimization formulation of a structural rigidity optimization methodology based on compliance with an initial design dependent stress field. This formulation leads to a convergent and very efficient optimization algorithm in the case of a local dependency of the initial stress with the optimization parameters.

Two cases are considered: topology optimization with an isotropic linear elastic composite material made of two isotropic linear elastic materials (one possibly being void) and orientational optimization with an orthotropic linear elastic material. We solve analytically in those two cases the minimization problem of local elastic energy with an initial stress field, with respect to material density in the case of topology optimization and with respect to orthotropy orientation in the case of orientational optimization.

Numerical examples for thermo-elastic stress loads are presented. Although the design domains and the boundary conditions are very simple,the obtained optimized density and orientation fields are quite complicated and the structural performance increase is demonstrated.

The main advantage of the methodology is the numerical efficiency thanks to local sensitivity analysis that are solved analytically or using a simple bisection procedure.

In the most general case of double minimization formulation of structural rigidity optimization based on compliance with an initial design dependent stress field, the minimization with respect to optimization parameters with a fixed total stress is global because of the global dependency of the initial stress with the optimization parameters. The optimization methodology is still 
valid but the sensitivity analysis recovers a numerical cost and the proposed method should be compared in that case to others in terms of numerical efficiency.

Allaire, G., Kohn, R.V., 1993. Optimal design for minimum weight and compliance in plane stress using extremal microstructures. Eur. J. Mech. ASolids 12, 839-878.

Allaire, G., Bonnetier, E., Francfort, G., Jouve, F., 1997. Shape optimization by the homogenization method. Num. Math. 76, 27-68.

Allaire, G., 2002. Shape optimization by the homogenization method. Springer Verlag, New York.

Ashby, M.F., 2006. The properties of foams and lattices. Phil. Trans. R. Soc. A. $364,15-30$.

Bendsøe, M.P., Kikuchi, N., 1988. Generating optimal topologies in structural design using a homogenization method. Comput. Meth. Appl. Mech. Eng. 71, 197-224.

Bendsøe, M.P., 1989. Optimal shape design as a material distribution problem. Struct. Opt. 1, 193-202.

Bendsøe, M.P., 1995. Optimization of structural topology, shape and material. Springer-Verlag.

Cheng, G., Pedersen, P., 1997. On sufficiency conditions for optimal design based on extremum principles of mechanics. J. of Mech. Phys. Sol. 34, $415-434$. 
Cho, S., Choi, J.-Y., 2005. Efficient topology optimization of thermoelasticity problems using coupled field adjoint sensitivity analysis method. Finite Elem. Anal. Des. 41, 1481-1495.

Desmorat, B., 2007. Structural rigidity optimization with frictionless unilateral contact. Int. J. Solids Struct. 44, 1132-1144.

Gao, T., Zhang, W., 2010. Topology optimization involving thermo-elastic stress loads. Struct. Multidisc. Optim. 42, 725-738.

Hashin, Z., Shtrikman, S., 1963. A variational approach to the theory of the elastic behaviour of multiphase materials. J. Mech. Phys. Solids 11, 127-140.

Jog, C., 1996. Distributed-parameter optimization and topology design for non-linear thermoelasticity. Comput. Meth. Appl. Mech. Eng. 132, 117134.

Levin, V.M., 1967. Thermal expansion coefficients of heterogeneous materials. Mekhanika Tverdoga Tela 2, 88-94.

Li, Q., Steven, G.P., Xie, Y.M., 2001. Thermoelastic topology optimization for problems with varying temperature fields. J. Thermal Stresses 24, 347366.

Pedersen, N.L., 2001. On topology optimization of plates with prestress. Int. J. Numer. Meth. Engng. 51, 225-239.

Pedersen, N.L., 2002. Topology optimization of laminated plates with prestress. Comput. Struct. 80, 558-570. 
Pedersen, P., Pedersen, N.L., 2010. Strength optimized designs of thermoelastic structures. Struct. Multidisc. Optim., 42, 681-691.

Rodrigues, H., Fernandes, P., 1995. A material based model for topology optimization of thermoelastic structures. Int. J. Numer. Meth. Engng. 38, 1951--1965.

Sigmund, O., Torquato, S., 1997. Design of materials with extreme thermal expansion using a three-phase topology optimization method. J. Mech. Phys. Solids 45, 1037-1067.

Zhou, M., Rozvany, G.I.N., 1991. The COC algorithm, Part II: topology, geometry, and generalized shape optimization. Comput. Meth. Appl. Mech. Eng. 89, 197-224. 


\begin{tabular}{|c|c|c|c|}
\hline Material & Aluminium & Titanium & Copper \\
\hline Density & $2700 \mathrm{~kg} / \mathrm{m}^{3}$ & $4510 \mathrm{~kg} / \mathrm{m}^{3}$ & $8940 \mathrm{~kg} / \mathrm{m}^{3}$ \\
\hline Bulk modulus & $73 \mathrm{GPa}$ & $120 \mathrm{GPa}$ & $134 \mathrm{GPa}$ \\
\hline Shear modulus & $26 \mathrm{GPa}$ & $41 \mathrm{GPa}$ & $46 \mathrm{GPa}$ \\
\hline Poisson's ration & 0.34 & 0.35 & 0.34 \\
\hline Thermal expansion coefficient & $22.8 * 10^{-6} \mathrm{~K}^{-1}$ & $9.0 * 10^{-6} \mathrm{~K}^{-1}$ & $16.8 * 10^{-6} \mathrm{~K}^{-1}$ \\
\hline
\end{tabular}

Table 1: Material parameters

\begin{tabular}{|c|c|c|}
\hline Young's Modulus & Poisson's Ration & Thermal Expansion coefficient \\
\hline $210 \mathrm{GPa}$ & 0.3 & $13 * 10^{-6} K^{-1}$ \\
\hline
\end{tabular}

Table 2: Steel material parameters

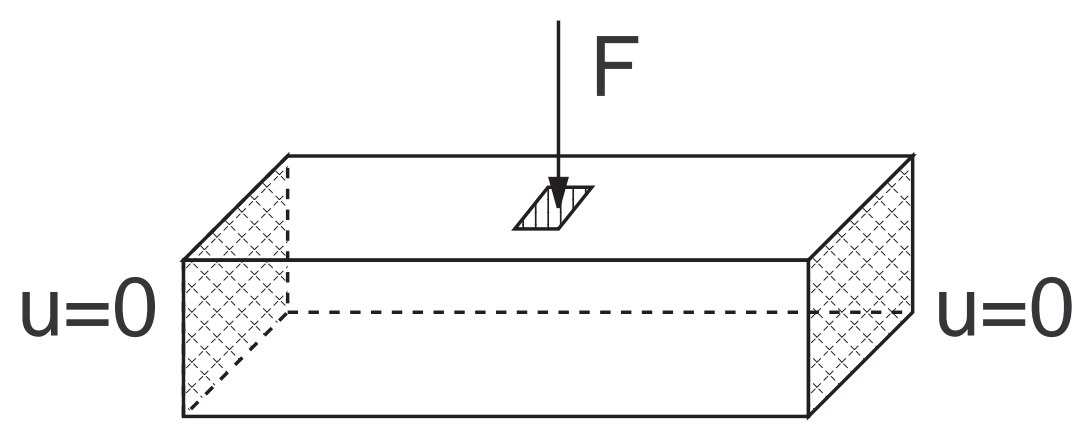

Figure 1: Beam example: geometry and boundary conditions 


\begin{tabular}{|c|c|c|c|c|}
\hline & \multicolumn{2}{|c|}{ NO thermo-elastic stress } & \multicolumn{2}{|c|}{ WITH thermo-elastic stress } \\
\hline & $\begin{array}{l}\text { Normalized } \\
\text { Compliance }\end{array}$ & $\begin{array}{l}\text { Normalized } \\
\text { maximal } \\
\text { displacement }\end{array}$ & $\begin{array}{l}\text { Normalized } \\
\text { compliance }\end{array}$ & $\begin{array}{l}\text { Normalized } \\
\text { maximal } \\
\text { displacement }\end{array}$ \\
\hline Initial (100\% volume) & 1 & 1 & 32.8 & 1.2 \\
\hline Optimal (20\% volume) & 4.6 & 4.3 & 8.4 & 4.1 \\
\hline
\end{tabular}

Table 3: Beam example: Normalized numerical results with respect to the initial state with NO thermo-elastic stress loads 


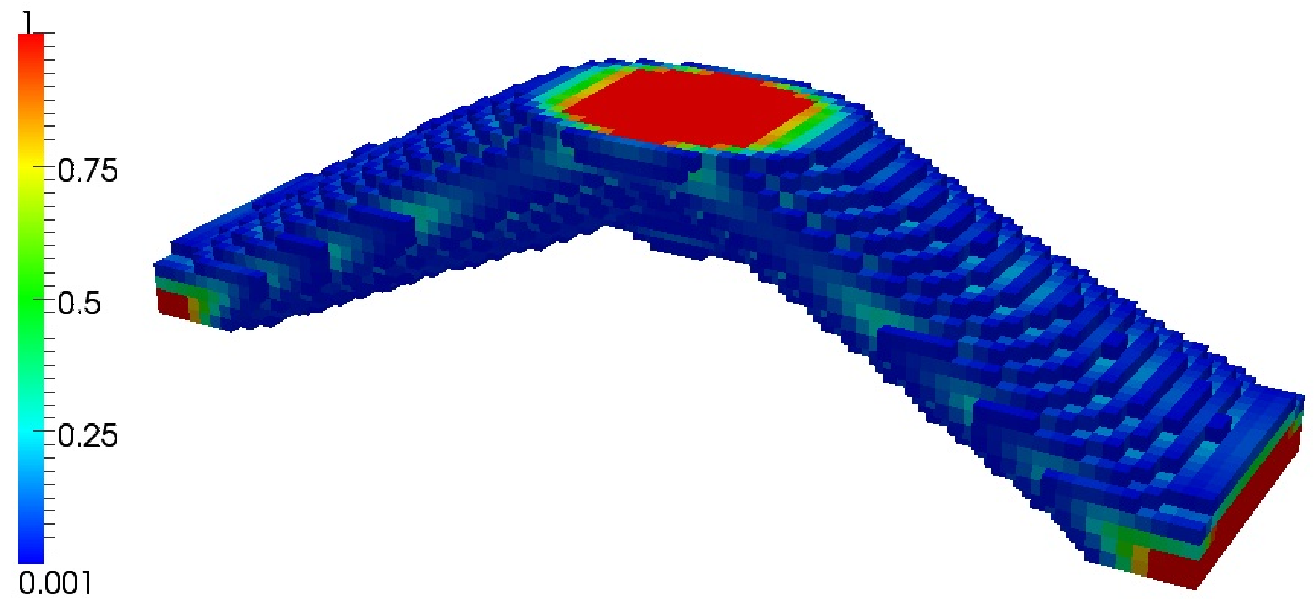

(a) $3 \mathrm{D}$ view with a density lower threshold equal to $10^{-2}\left(\rho_{\min }=10^{-3}\right)$

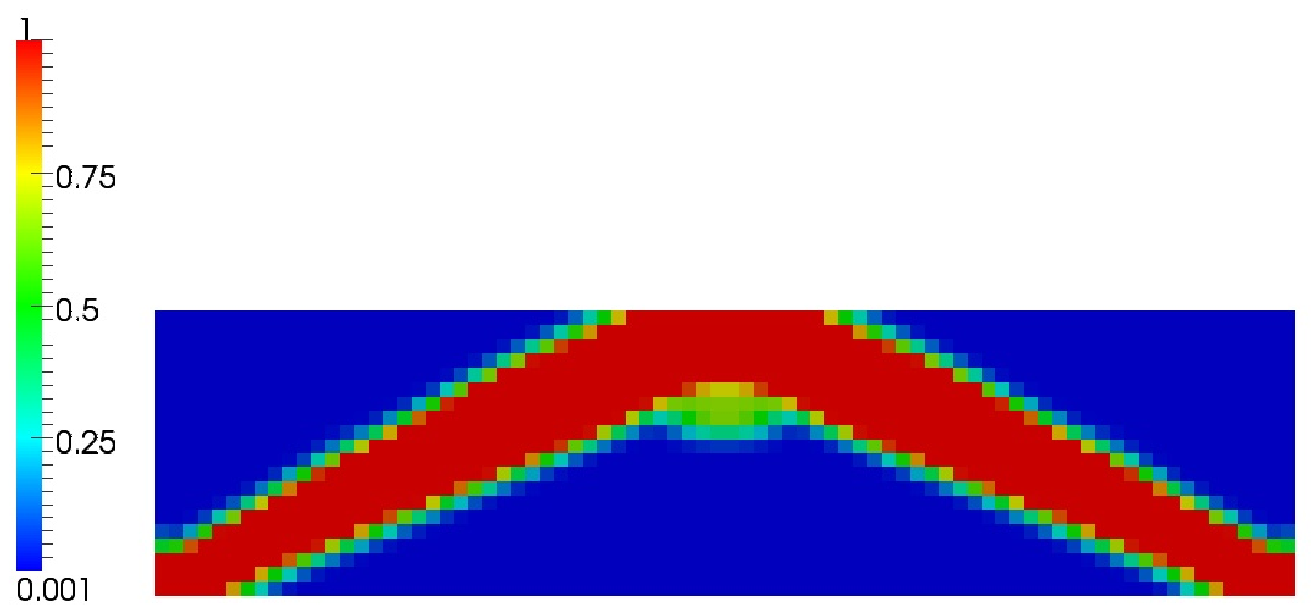

(b) $2 \mathrm{D}$ view of the central vertical section

Figure 2: Optimal density distribution without thermo-elastic stress load 


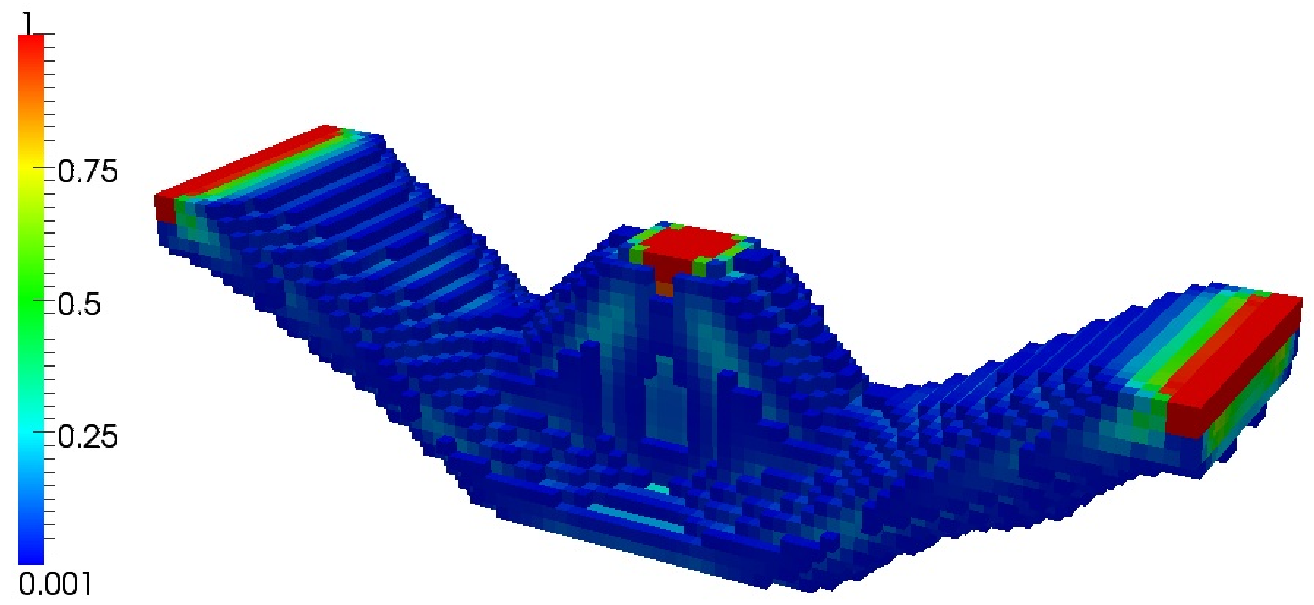

(a) $3 \mathrm{D}$ view with a density lower threshold equal to $10^{-2}\left(\rho_{\min }=10^{-3}\right)$

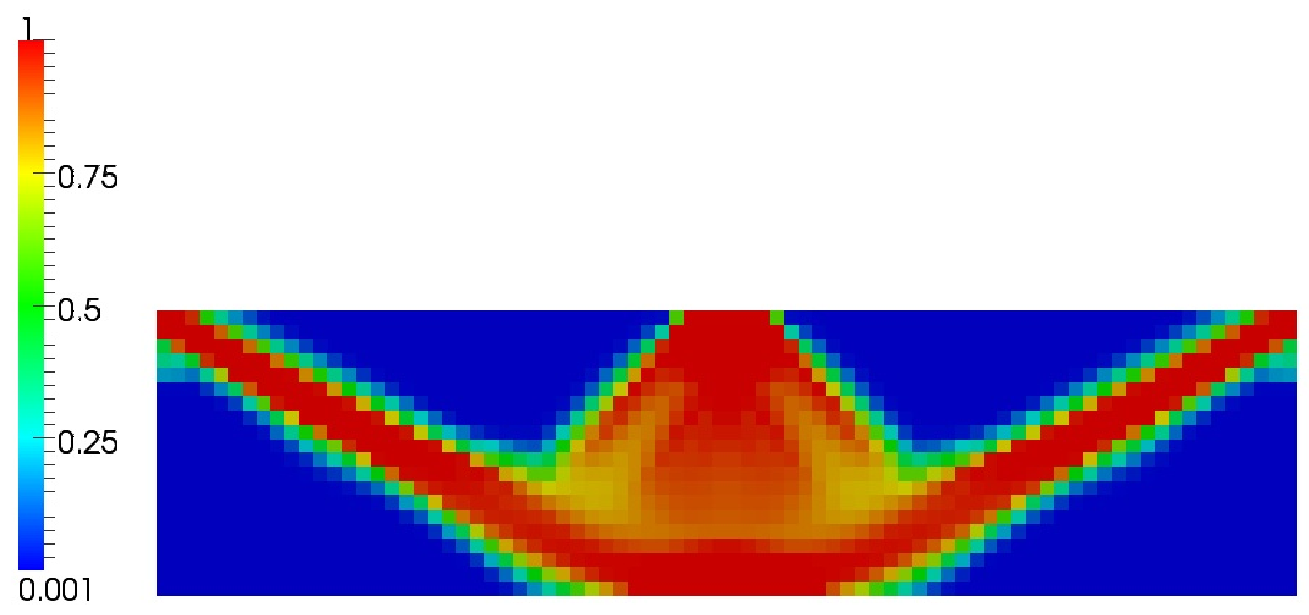

(b) $2 \mathrm{D}$ view of the central vertical section

Figure 3: Optimal density distribution with thermo-elastic stress load $\left(T=-50^{\circ} \mathrm{C}\right)$ 


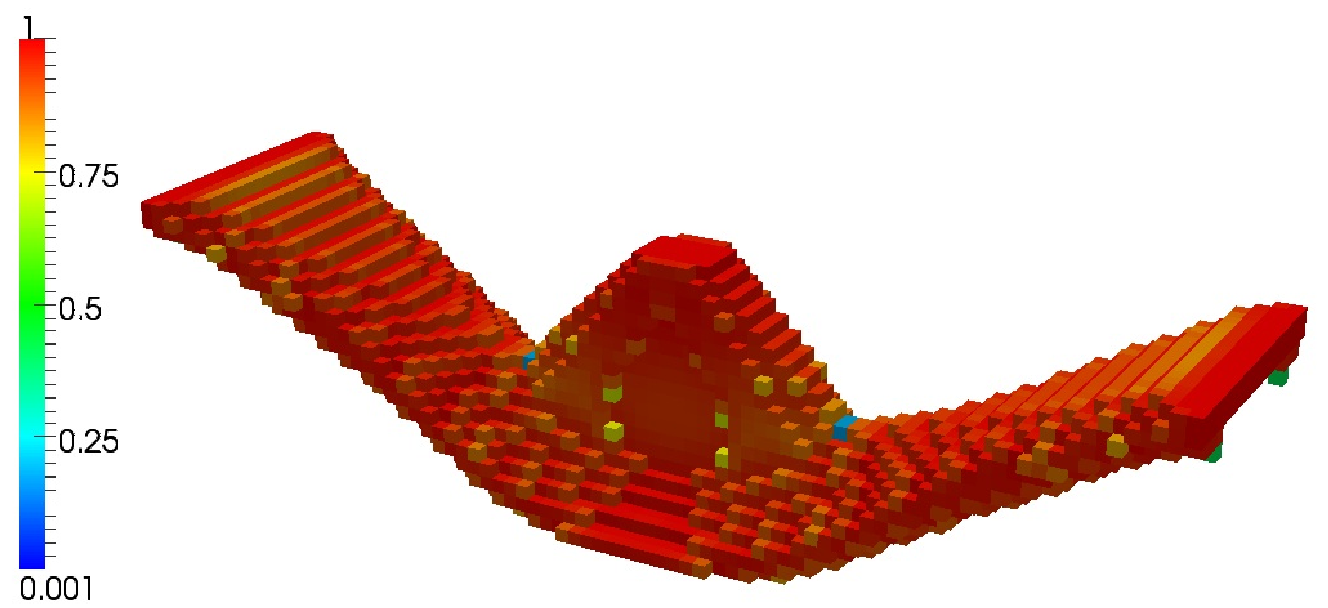

(a) $3 \mathrm{D}$ view with a density lower threshold equal to $10^{-2}\left(\rho_{\min }=10^{-3}\right)$

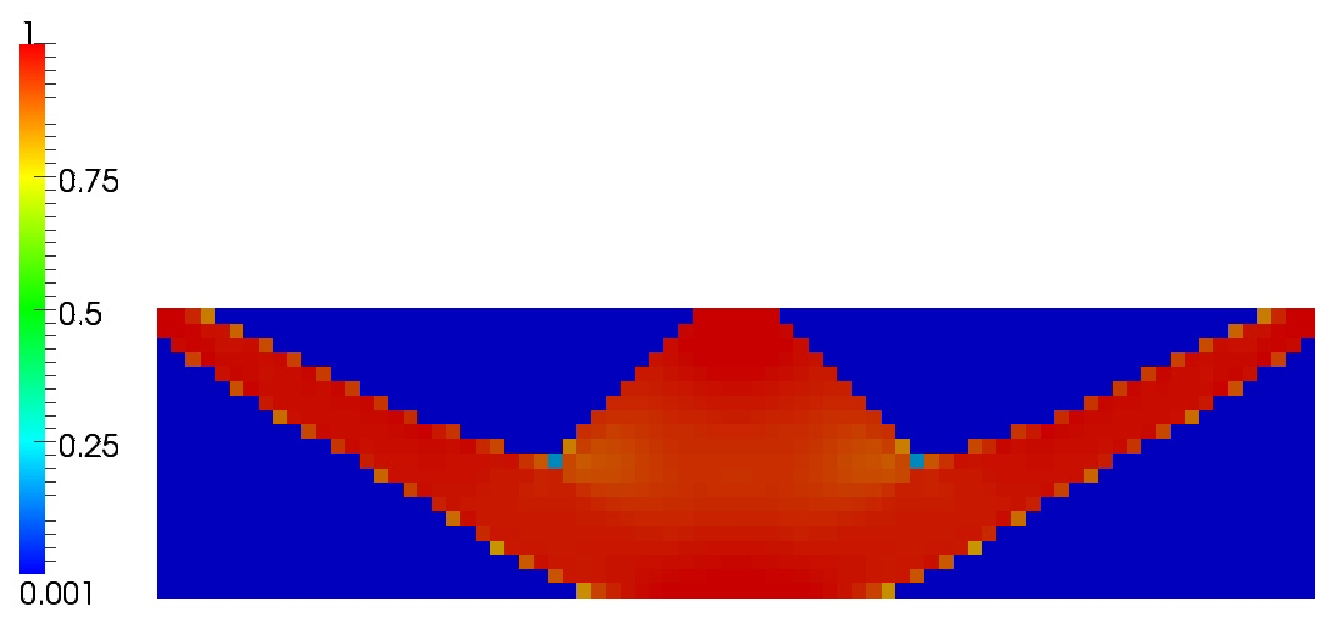

(b) $2 \mathrm{D}$ view of the central vertical section

Figure 4: Penalized optimal density distribution with thermo-elastic stress load $(T=$ $\left.-50^{\circ} \mathrm{C}\right)$ 


\begin{tabular}{|c|c|}
\hline Longitudinal Young's Modulus & $85000 \mathrm{MPa}$ \\
\hline Transverse Young's Modulus & $5600 \mathrm{MPa}$ \\
\hline Shear Modulus & $2100 \mathrm{MPa}$ \\
\hline Poisson's ration & 0.34 \\
\hline Longitudinal thermal expansion coefficient & $-1 * 10^{-6} \mathrm{~K}^{-1}$ \\
\hline Transverse thermal expansion coefficient & $70 * 10^{-6} \mathrm{~K}^{-1}$ \\
\hline Isotropic thermal conductivity & $0.2 \mathrm{Wm}^{-1} \mathrm{~K}^{-1}$ \\
\hline
\end{tabular}

Table 4: Aramid/Epoxy composite material parameters

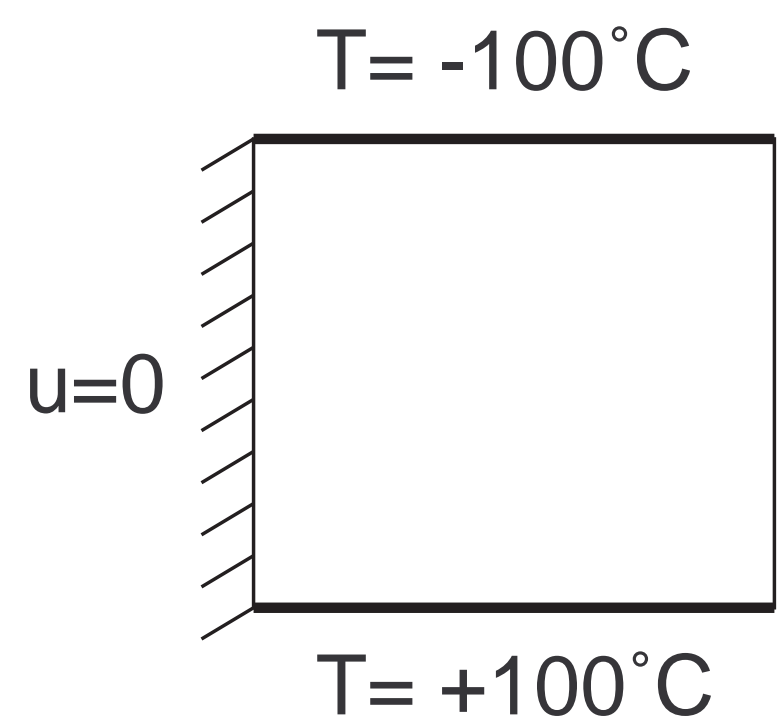

Figure 5: Composite plate example: geometry and boundary conditions 


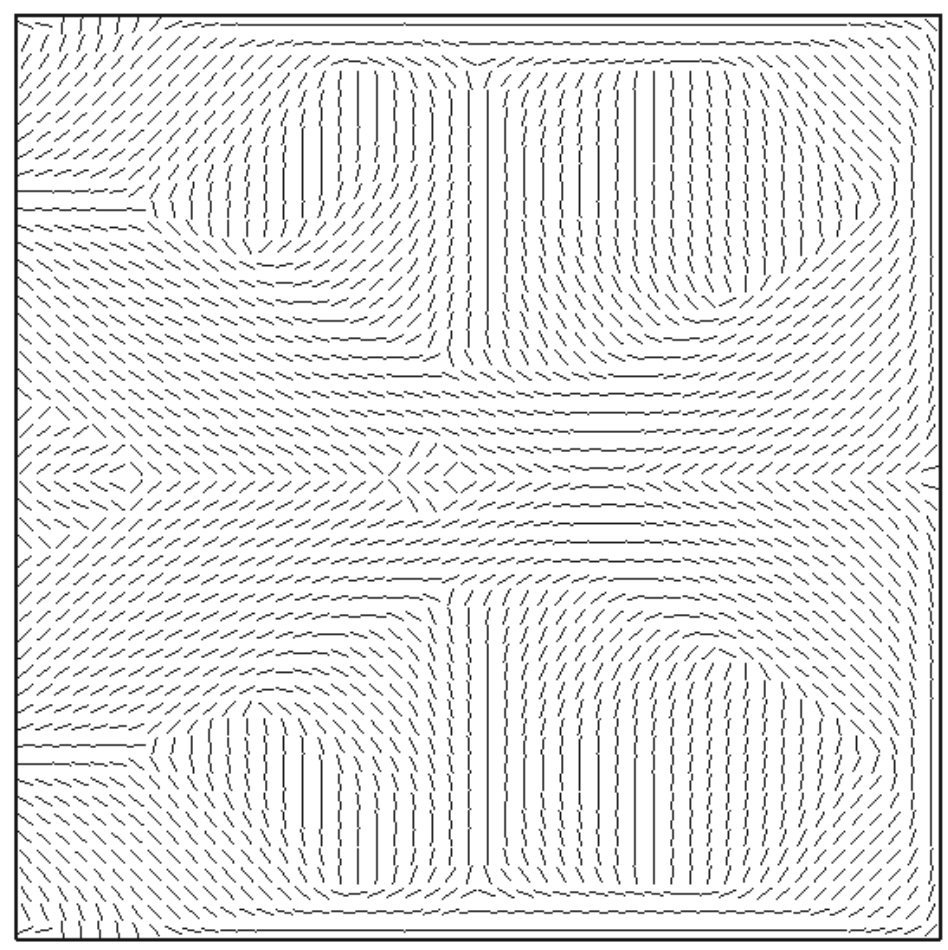

Figure 6: Optimal material orthotropy orientation 


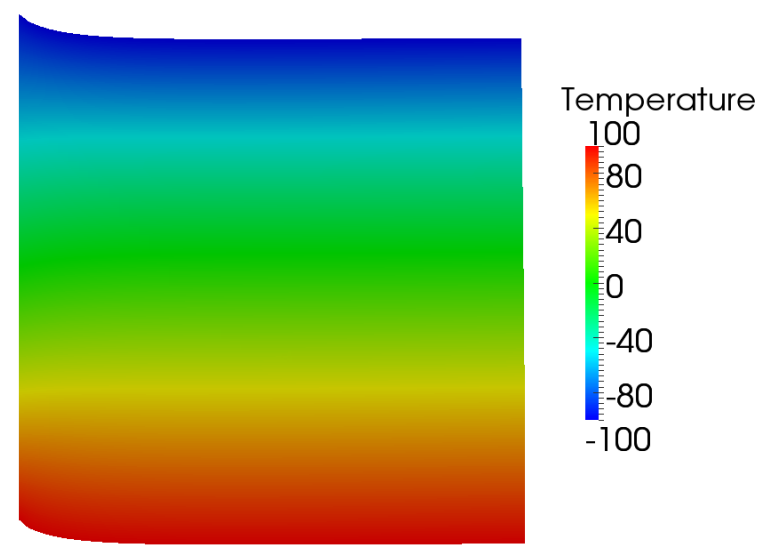

Figure 7: Initial deformed configuration (amplification factor x30) with temperature field

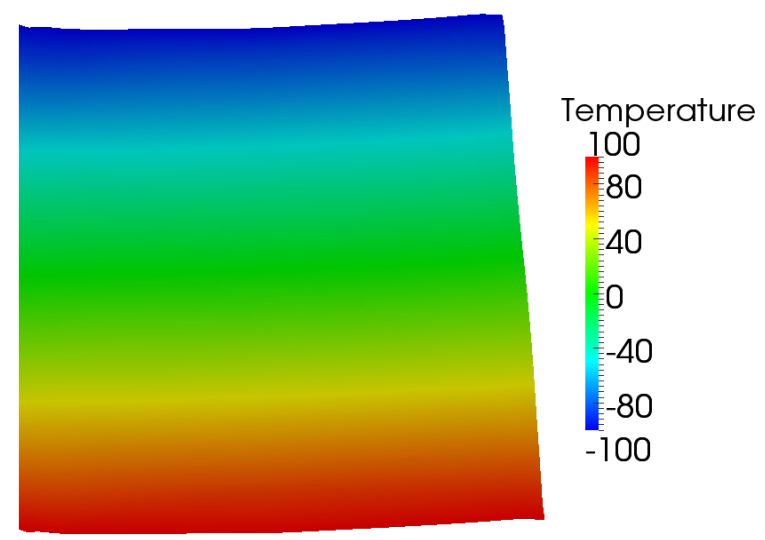

Figure 8: Optimal deformed configuration (amplification factor $\mathrm{x} 30$ ) with temperature field 